\title{
Narrow QRS tachycardia with atrial and ventricular cycle length wobbling - What is the mechanism?
}

Shunmuga Sundaram ${ }^{1}$ and Vidhya Ganesan ${ }^{1}$

${ }^{1}$ Velammal Medical College Hospital and Research Institute

May 6, 2020

Title Page

Narrow QRS tachycardia with atrial and ventricular cycle length wobbling - What is the mechanism?

Author Details

Corresponding Author:

Dr. Shunmuga Sundaram Ponnusamy MD., DM., PDF (EP)., CEPS

Assistant Professor, Department of Cardiology

Velammal Medical college hospital and research institute

Madurai, Tamilnadu, India

Additional Authors:

Dr. Vithiya Ganesan MBBS., MD.,

Associate professor, Department of Microbiology Velammal Medical College hospital and research institute Maduria, Tamilnadu, India

\section{Address for correspondence}

Dr P. Shunmuga Sundaram MD., DM., PDF (EP)., CEPS

Assistant Professor, Department of Cardiology

Velammal Village, Airport ring road

Velammal Medical college hospital and research institute

Madurai - 625009

Mobile no: +919444712846

Email id: shunmuga.pgi@gmail.com

Word Counts: 958 words

Financial Source

No funding or financial sources received for this study

We have no conflicts of interest to disclose .

Narrow QRS tachycardia with atrial and ventricular cycle length wobbling - What is the mechanism? 


\section{Case Presentation}

A 41 years old lady presented to us with drug refractory narrow QRS tachycardia. Echocardiography revealed normal left ventricular systolic function. 12 lead electrocardiography (ECG) showed long RP (RP $>$ PR) narrow QRS tachycardia. Radiofrequency catheter ablation was performed after obtaining informed consent. Intracardiac electrograms and 12 lead ECG were continuously monitored using Workmate Claris system (Abbott, Plymouth, MN). Upper limb Venous angiography showed dilated coronary sinus with left sided superior vena cava. Basal AH and HV intervals were $85 \mathrm{~ms}$ and $44 \mathrm{~ms}$ respectively. Patient had spontaneously inducible tachycardia (Fig 1B) which could be terminated by intravenous adenosine. ECG showed narrow QRS tachycardia with variation in both atrial and ventricular cycle length (Fig 1A). Intracardiac electrogram during tachycardia showed variation in $\mathrm{H}-\mathrm{H}, \mathrm{A}-\mathrm{A}, \mathrm{V}-\mathrm{V}$ and $\mathrm{HA}$ intervals (Fig 2)

1. What is the mechanism of the tachycardia onset?

2. What is the substrate for re-entry?

3. What could be the reason for the variation in $\mathrm{A}-\mathrm{A}, \mathrm{H}-\mathrm{H}, \mathrm{H}-\mathrm{A}$ and $\mathrm{V}-\mathrm{V}$ intervals?
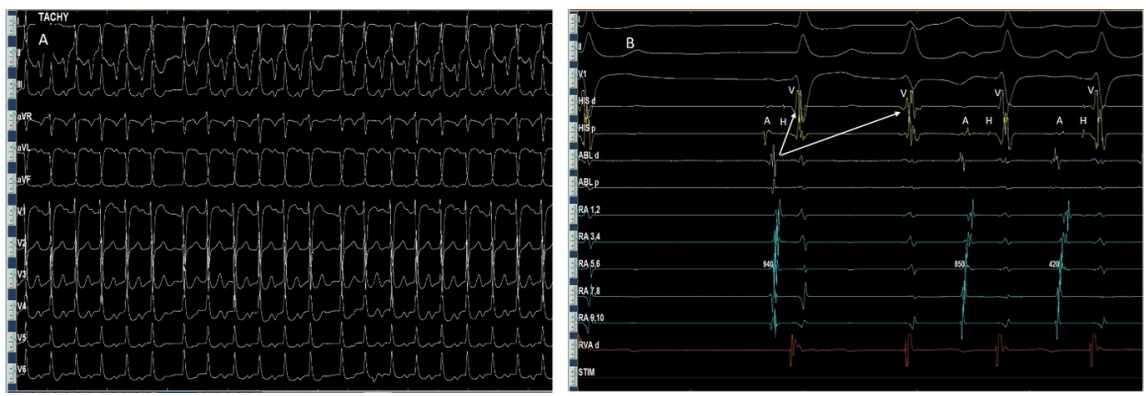

Fig 1: A -12 lead ECG showing narrow QRS tachycardia with varying RP interval. B - Electrogram showing the spontaneous initiation of tachycardia. Single atrial impulse has got conducted through both fast and slow pathways to produce 1:2 AV response followed by initiation of tachycardia. His d and His $p-H i s$ electrograms - distal and proximal, ABL $d$ and $P$ - Ablation catheter distal and proximal, RA 1-10 - Coronary sinus electrograms, $R V A d$ and $p-R V$ electrogram distal and proximal
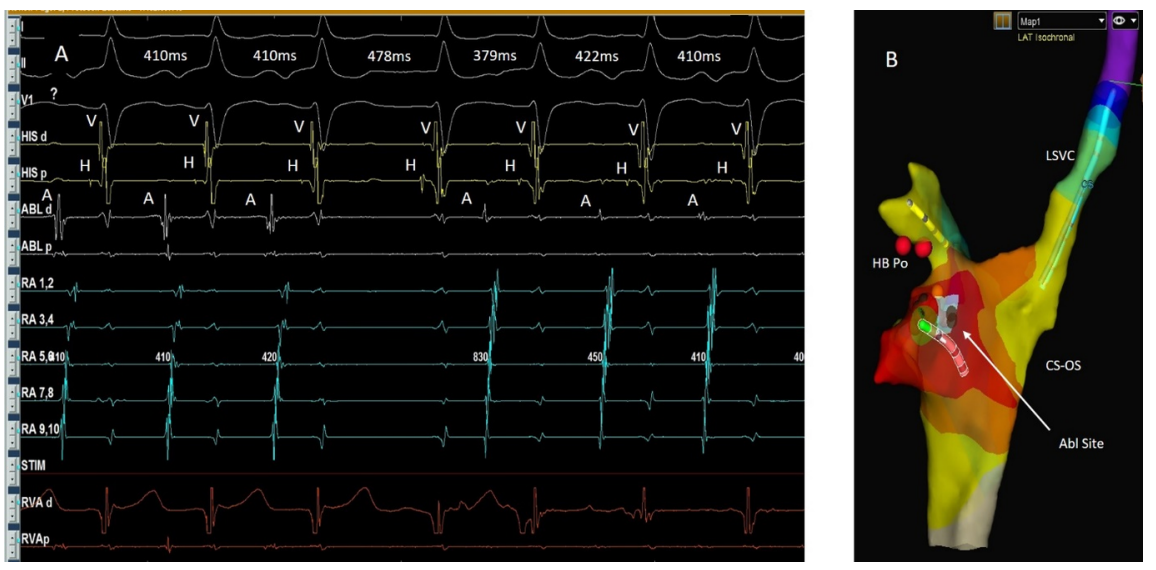

Fig 2: $A$ - Intracardiac electrogram showing variation in $A-A, H-H, H-A$ and $V$ - $V$ intervals due to decremental conduction in upper common pathway and antegrade slow pathway (S1). B- Electroanatomic map showing dilated coronary sinus ostium (CS-OS) with left SVC (LSVC) and the site of earliest atrial activation (white spot). His $d$ and His $p$-His electrograms - distal and proximal, ABL $d$ and P-Ablation catheter distal and 
proximal, RA 1-10 - Coronary sinus electrograms, RVA $d$ and $p-R V$ electrogram distal and proxiamal, His po- His potential

Commentary:

12 lead ECG showed narrow QRS tachycardia with retrograde VA wenkebaching, hence Atrio-ventricular reciprocating tachycardia (AVRT) and atrial tachycardia (AT) could be excluded. Intracardiac electrogram during spontaneous induction showed classical 1:2 AV nodal conduction followed by initiation of tachycardia (Fig 1B). Several features favor AV nodal re-entrant tachycardia (AVNRT) in this case: VA dissociation, response to adenosine, 1:2 AV nodal conduction, concentric atrial activation pattern and fixed HV interval (44ms) during tachycardia. The possibility of automatic junctional tachycardia was excluded based on response to premature atrial extrastimuli and intravenous adenosine. Intracardiac electrogram showed variation in A-A, V-V, H-H and HA intervals. Atypical AVNRT of slow - slow type with decremental conduction of upper common pathway (UCP) could explain the variation in A-A and H-A intervals (Fig 3A, B and C). The $\mathrm{H}-\mathrm{H}$ and $\mathrm{V}-\mathrm{V}$ interval variations could be explained by presence of multiple slow pathways as below

1. Three slow pathways with different electrophysiological properties

2. Initial re-entry between slow pathway-1 (S1-antegrade) and slow pathway-2 (S2-retrograde) at a cycle length of $410 \mathrm{~ms}$ with concealed conduction into slow pathway-3 (S3) and decremental conduction in upper common pathway (fig 3A)

3. Decremental conduction of S1 resulting in gradual prolongation of $\mathrm{H}-\mathrm{H}$ interval followed by block which favored the transient re-entry between S3 (antegrade) and S2 (retrograde) (cycle length $379 \mathrm{~ms}$ ) and subsequent resumption of $\mathrm{S} 1$ conduction (fig 3B \& C)

Fig 3: A - Atypical slow- slow AVNRT with re-entry between S1 and S2 with concealed conduction into S3. Upper common pathway showed decremental conduction. B-Transient block in $S 1$ due to decremental conduction favored re-entry between S3 and S2 with concealed conduction into S1. C - Ladder diagram showing the same phenomenon. The decremental conduction in $S 1$ prolonged $H-H / V-V$ interval from $410 m s$ to $478 \mathrm{~ms}$. S3 could conduct in the next beat with $H-H$ interval of $379 m s$ as $S 1$ was transiently blocked. In the subsequent beat $S 1$ conduction resumed with minimal prolongation of $H$ - $H$ interval due to concealed conduction. Atrial conduction occurred decrementally through upper common pathway (UCP). S1,2,3-slow pathways 1,2 and 3, F-fast pathway, LCP-Lower common pathway The possibility of two antegrade slow pathway conduction has been described previously as a cause for cycle length alteration during AVNRT ${ }^{1}$. Multiple slow pathways are required for the initiation of re-entrant tachycardia after 1:2 AV response which otherwise would not have induced. In our patient the cycle length alteration was noted in both atrium $(\mathrm{A}-\mathrm{A})$ and ventricle $(\mathrm{V}-\mathrm{V})$. This could be explained by the rare combination of decremental conduction in both upper common pathway and antegradely conducting slow pathway (S1). The second pathway (S2) has different electrophysiological properties (conduction velocity and refractory period) as evidenced by the intracardiac electrogram and it is unlikely to be a fast pathway as the HA interval is long. The fourth beat in the figure $2 \mathrm{C}$ has slightly longer cycle length $(422 \mathrm{~ms})$ due to concealed conduction of previous impulse into S1. These findings emphasize the fact that both atrium and the ventricle are not the part of circuit in AVNRT. Electroanatomic mapping (ENSITE Velocity, Abbott, Plymouth, MN) confirmed earliest atrial activation at coronary sinus ostium (fig 2B) Slow pathway was ablated (medium curve catheter; 60W, 600C) which rendered the tachycardia non-inducible. Since there were three slow pathways involved in this patient, this rare variety could be labelled as atypical slow-slow-slow AVNRT. The mechanism of re-entry in typical as well as atypical AVNRT remains elusive ${ }^{2}$. There has been electrophysiologic evidence of multiple superior atrial inputs to the AV node ${ }^{3}$ that could explain multiple sites of early atrial activation during tachycardia. Cycle length alternans can occur during AVNRT due to either antegrade conduction via two slow pathways or junctional bigeminism ${ }^{4}$. The decremental conduction properties of antegrade slow pathway (S1) and upper common pathway were the reasons for variation in A-A, H-H, H-A and V-V intervals in our case of atypical slow-slow-slow AVNRT.References

1. Maury P, Raczka F, Piot C, Davy JM. QRS and cycle length alternans during paroxysmal supraventricular tachycardia: What is the mechanism? J Cardiovasc Electrophysiol 2002;13:92-3 
2. Katritsis DG, Camm AJ. Atriovenricular nodal reentrant tachycardia. Circulation 2010;122:831-40.

3. Wu J, Wu J, Olgin J, Miller JM, Zipes DP. Mechanisms underlying the reentrant circuit of atrioventricular nodal re-entrant tachycardia in isolated canine atrioventricular nodal preparation using optical mapping. Circ Res 2001;88:1189-95.

4. Surawicz B, Fisch C. Cardiac Alternans: Diverse mechanisms and clinical manifestations. J Am Coll Cardiol 1992;20:483-499
